\title{
The development and promotion of Electronic Theses and Dissertations (ETDs) within the UK
}

\section{Susan Copeland}

\author{
Andrew Penman
}

An increasing number of universities are accepting and encouraging the submission of theses and dissertations in electronic format. Two hundred and three institutions are now members of the international 'Networked Digital Library of Theses and Dissertations' (NDLTD), and in May 2003 over 200 individuals travelled to Berlin to participate in the ETD symposium ('Next Steps - Electronic Theses and Dissertations Worldwide'). The support of UNESCO and the work undertaken by key institutions such as Virginia Tech. has led to wide scale developments at national and individual level. Within the UK, funding from the Joint Information Systems Committee (JISC) has enabled three project teams to engage in research and development associated with the creation, management, and use of electronic theses and dissertations (ETDs).

This paper considers recent ETD-related activity in the UK within the broader international context. It concentrates, in particular, on the work of the Electronic Theses project consortium that is led by The Robert Gordon University. The benefits of ETDs, issues of concern, the selection of software, and advocacy requirements are amongst the topics addressed. The authors welcome comments on both the project and the information available on the Electronic Theses project Web pages.

\section{INTRODUCTION}

In recent years, numerous organisations, institutions and individuals have endeavoured to take advantage of I.T. developments to improve access to scholarly publications. Theses and dissertations are amongst the categories of material that benefit significantly from the opportunities that are now available for publishing in electronic format. Statistics from Virginia Polytechnic and State University (Virginia Tech.) in the USA reveal a very high level of use of electronic theses and dissertations (ETDs) that are available on the Web (1). An analysis of the accesses from U.S. domains reveals that use of these theses is not limited to educational establishments; over the past few years government, commercial and other miscellaneous organisations have consulted the works. In the 1990s, Virginia Tech. was one of the first institutions to encourage the creation of ETDs. 
Since then, the ETD movement has flourished internationally, and membership of the 'Networked Digital Library of Theses and Dissertations' (NDLTD), which was initiated in 1997, has increased significantly (2).

\section{UK BACKGROUND}

Within the UK, initial interest in ETDs, in the mid 1990s, led to the establishment of the 'UK Theses Online Group' (UTOG). The main aim of UTOG was to promote informed discussion on the topic of e-theses, with a view to persuading librarians, academic staff, research students and potential users of the end product to accept theses in non print formats. Between 1994 and 1998 UTOG considered issues associated with the production of theses as electronic documents, retrospective digitisation, and the use of ETDs. In order to gain an understanding of patterns of production and use of doctoral theses in the UK, UTOG conducted a survey involving over 2000 PhD students, 1740 supervisors of higher degree students, and 125 librarians. The funding required to undertake this survey, which was based upon postal questionnaires, was provided by the British Library and the Joint Information Systems Committee (JISC). The results of the survey were published in a British Library report and made available on the University of Edinburgh Web pages (3). The findings were also discussed at a seminar in London in 1997.

The work undertaken by UTOG provided a good basis upon which later projects could build. The survey revealed that most researchers found it useful to consult theses as part of their literature searches. Levels of use did not appear to be monitored adequately to provide clear statistics, but librarians reported that many theses in their collections were not used. This situation suggested that improved access to theses would both benefit researchers and increase the exploitation of valuable resources held by academic libraries. Furthermore, the survey results indicated that, by this time, the majority of theses were produced 
initially in electronic format (i.e. word processed using a standard package) and most students were positive about the idea of their work being made available on the Web.

UTOG achieved its goals. The group gained an understanding of patterns of theses production and use, and the findings were publicised with a view to encouraging a culture change amongst those associated with the PhD process. However, without a steady source of funding, it became difficult for UTOG to continue its activities and from 1998 onwards promotional work to maintain interest in the development of ETDs in the UK became restricted to the efforts of a few enthusiastic individuals.

\section{THE INTERNATIONAL SCENE}

While ETD activities in the UK were limited, the period around the turn of the century witnessed significant developments internationally. In September 1999 a workshop was held at the UNESCO headquarters to review existing ETD projects, to identify the needs of developing countries, and to consider potential international action in this area (4). Since this time, UNESCO has continued to support developments associated with ETDs, and has produced informative documentation such as: 'The Guide for Electronic Theses and Dissertations' (5).

Virginia Tech. has continued to provide an important role by co-ordinating many of the international developments and promoting the work of the members of the NDLTD. A key promotional activity is the annual ETD Symposium. The 2003 ETD Symposium, 'Next Steps - Electronic Theses and Dissertations Worldwide' was held at Humboldt University in Berlin (6). This was the first time the conference had been held outside the USA and it proved a great success. Over 200 participants from a wide range of countries attended and the discussions were both wide ranging and stimulating. Confidence in the annual event is such that 
planning is already underway for the 2005 event in Sydney that will follow the 2004 Symposium 'Distributing knowledge worldwide through better scholarly communication' in Kentucky (7).

Contributions to the annual symposium, together with the wealth of information available on individual university ETD Web pages, reveal the diverse nature of the issues associated with the creation, management and use of electronic theses. These range from the means of addressing faculty resistance to 'born digital' theses, and concern about the I.T. skills needed in order to incorporate multimedia, to the legal issues associated with copyright and IPR and the political implications of establishing ETD consortia and national networked ETD libraries. Technical advances continue to open up new possibilities, and issues associated with security and archiving continue to pose problems. However, with close collaboration at global level, a considerable amount of progress has been made within the last few years and much is being learned through the sharing of experience.

The French language CyberThèses (8), the Australian Digital Theses Program (ADT) (9), and the Consorci de Biblioteques Universitaries de Catalunya (10) illustrate the range and scale of the collaborative ETD projects that are now underway. Some projects are concentrating on accumulating content; others are dealing with more specific issues. For example, amongst other work, the CERIST Research Centre in Algiers (11) is investigating long-term conservation and preservation, and Humboldt University in Berlin (12) has undertaken research in the area of workflow models. Universities that have required ETDs for some time have gained valuable experience in solving various problems that have arisen and many of the universities in the USA, in particular, have provided useful advice on their Web pages. 
Members of the NDLTD are based in a wide variety of countries and operate within very different cultures. The theses produced at the different institutions vary in a number of ways, for example in terms of length and style. In some countries the PhD is based partly on articles that have been published already in journals. By reviewing the information provided by a diverse range of universities, it is possible to obtain a wealth of ideas that can be adapted to fit the needs of organisations that are in the early stages of developing ETD collections.

\section{THE JISC 'FAIR' PROGRAMME}

\section{'FAIR' projects}

In the UK, despite the demise of UTOG, interested individuals have kept abreast of activities underway in other countries. In 2002, development work recommenced when a significant amount of funding was made available from the Joint Information Systems Committee (JISC) as part of the 'Focus on Access to Institutional Resources' (FAIR) Programme (13). This programme involves members of the Higher and Further Education community in the UK and, in keeping with the vision of the 'Open Archives Initiative' (OAI) (14), its aim is to encourage the disclosure of institutional assets and to further understanding about associated technical, organisational and cultural challenges. Fourteen projects were successful in gaining funding as part of the FAIR programme and, of these, three directly involve ETDs:

- DAEDALUS (15) - The DAEDALUS project (Data providers for Academic Econtent and the Disclosure of Assets for Learning, Understanding and Scholarship) is based at the University of Glasgow. It is building a range of OAI-compliant digital collections. The project is investigating the use of three different software packages to sort various categories of material, including pre-prints, post-prints, administrative documents and ETDs. It is considering both technical and cultural issues associated with this work. 
- Theses Alive! (16) - This project is based at the University of Edinburgh. It is developing an OAI-compliant thesis submission system and a system for the management of ETDs that institutions publish on the Web.

- Electronic Theses (17) - The Electronic Theses project is led by The Robert Gordon University in Aberdeen. It involves a number of partner institutions: The British Library, the University of Aberdeen, Cranfield University and the University of London Library. The project members are investigating issues associated with the production, management and use of electronic theses, including those that are 'born digital', and they are aiming to produce a model which can be adopted at national level.

\section{The 'Electronic Theses' project}

The Electronic Theses project received funding initially from JISC for the period July 2002 to July 2004 to fulfil the aim of "evaluating a wide range of existing practices of e-theses production, management and use against a set of criteria in order to produce models for use within the UK information environment". Additional funding was made available for a second phase of the project, for the period October 2003 to September 2004, to allow the project team "To develop e-theses in a service environment". Furthermore, The Robert Gordon University made internal funding available for the project to take account of the needs of such an independent repository within a broader Virtual Research Environment (VRE).

The specific objectives of the project are as follows:

Phase 1

- To identify examples and recurrence of relevant best practice which could be adopted throughout the UK information environment. 
- To evaluate a comprehensive selection of methods, which are used internationally to create, store, organise, manage and access electronic theses.

- To liaise with members of the DAEDALUS and Theses Alive! project teams to ensure that there is co-ordination within the E-Theses Cluster.

- To produce a model, or models, of e-theses production, which could be recommended for converting paper-based theses into digital format.

- To produce a model or models, of e-theses production which could be recommended for creating born-digital theses in a format that allows increased opportunities for authors to express their research in more dynamic and media-rich ways.

- To produce a set of guidelines for the storage, organisation and management of the above collections.

- To produce models for access arrangements to the above, taking into account the advantages and disadvantages of centralised, federated, distributed or individual collections.

- To attend relevant conferences and organise a seminar for the purposes of networking, sharing expertise and disseminating research findings.

Phase 2

- To create an e-theses repository hosted using EPrints software.

- To create an e-theses repository hosted using DSpace software.

- To demonstrate the integration of the above within institutional WebPages. 
- To assess the difference between the capabilities of EPrints and DSpace archives from the point of view of installation, administration and usage.

- To identify preferred methods of embedding audio-visual resources and interactive material within a single ETD file.

- To identify preferred methods of delivery of e-theses at institutional and national level.

Information on the progress achieved in each of the above areas is available on the project Web pages. Guidelines are being produced to assist universities in the UK which wish to establish ETD repositories. Attention is being given to promoting the idea of electronic theses to research students, research supervisors, and university administrators as well as to library staff.

\section{Promotional work}

Within RGU, advocacy work to achieve a culture change with regard to attitudes towards ETDs targeted various levels of staff. Library staff were kept informed of international developments in this area and the plans for their own institution, academic staff were invited to a seminar to encourage them to appreciate the advantages offered by ETDs, and members of the senior management were approached to identify the policies and procedures that would need to be amended. The Registry was consulted to ensure that University regulations relating to the submission of theses were updated appropriately. Papers were presented to the Research Committee to allay fears about plagiarism and to confirm that there would be an option for students to place an embargo on the publication of their thesis on the Web for a specific period if they wished to pursue a patent application etc. 
Each institution has its own committee structure and regulations relating to the submission of theses and dissertations. Early planning is necessary in order to ensure that the appropriate changes are in place to support a new theses submission process. Research supervisors need to be enthused in order to give support to the concept of ETDs, administrative staff need to be assured that appropriate processes have been put in place, and students need to see ETDs as an opportunity to express their results creatively and to have their work publicised at international level.

In addition to ensuring that arrangements are in place at institutional level, it is important to ensure that the aims of local consortia or national activities and plans are taken into account when establishing an ETD collection. In the UK, a major seminar was held in London in January 2004 to update the academic and library community on the progress and proposed recommendations resulting from the JISC-funded ETD projects and to obtain the views of those who would be likely to be involved in developments in this area in future. The event was fully booked within a few days of being advertised, and feedback indicated that a significant number of individuals and institutions are keen to begin building a collection of electronic theses. The majority of those who provided feedback from the seminar confirmed the views expressed in responses to an earlier questionnaire that had been sent out on the lis-SCONUL mailing list to chief librarians in the UK i.e. that they were interested in establishing an institutional e-theses repository and that they would appreciate guidance on how to do this. They were also keen on the idea of the creation of a 'national hub' from where theses from institutions throughout the country would be made available in electronic format. If such a hub is established it will give smaller institutions the option of contributing to this rather than setting up their own repository. This may be an appealing option if the latter exercise appears too costly or too intensive in terms of staff time. 
The situation in Wales varies somewhat from the rest of the UK as the National Library currently holds printed copies of selected theses of relevance to Wales. To avoid unnecessary duplication of effort and resources, arrangements will need to be made to accommodate the needs of those storing and those accessing these theses within a framework for the development of ETD collections throughout the UK. To this end, plans are underway for interested parties to attend a seminar in Aberystwyth in September 2004 with a view to developing a strategy for the future. The findings from this event will feed into another seminar being organised by the Electronic Theses project group which will be held at The British Library in London later that month. The latter event will include final details of the proposed model for the UK.

The London seminar in January 2004 covered topics as diverse as workflow issues, policies and procedures, a draft metadata core set, submission software and ETD collections as part of broader institutional repositories. Slides from the seminar, and details about the metadata core set, are available on the Electronic Theses project Web pages. Similarly, the papers that will be presented at the September 2004 seminar will also be made available to a wide readership via Web pages.

\section{GENERAL ISSUES}

Despite the wide variation in the issues being addressed by the organisations worldwide that are involved in ETD development work, several key factors are common to all. In particular, there must be an understanding of the benefits to be gained from the work, and these benefits must be conveyed to both the content providers and the potential users of the service. There must also be an appreciation of the areas of concern, and a willingness to share ideas about possible solutions in order to make progress as quickly as possible. 


\section{Benefits}

\section{Benefits for students}

The creation of theses and dissertations in electronic format suitable for inclusion in an institutional or national repository may involve students in a small amount of additional work. However, the benefits are significant.

- Students may take advantage of the opportunity to express their research results in more flexible and creative ways.

- Students' theses are likely to be read more widely if they are easily accessible via the Web.

- ETDs are cost effective where the alternative is for students to provide multiple copies of bound paper theses.

- The process of creating ETDs improves students' IT skills.

Benefits for faculty/academic staff

- Researchers are able to obtain immediate access to theses 24 hours a day.

- Researchers are able to obtain remote access to theses.

- Simultaneous use by a number of researchers is possible.

- The availability of the full text of theses obviates the need for researchers to judge potential relevance from abstracts and eliminates the time and costs involved in requesting full-text hard copies on inter-library loan.

- Research supervisors can encourage their students to produce their research findings in more diverse and more appropriate ways.

- Research supervisors can access theses from other institutions easily in order to compare and contrast styles and standards of student work. 


\section{Benefits for institutions}

- Institutions benefit from improved access to the research findings of their staff and students. Increased levels of use of the material produced at a particular university can help to promote the research profile of the institution.

\section{Benefits for libraries}

Libraries also benefit when theses and dissertations are made available in electronic format since:

- $\quad$ storage space is saved;

- a saving is made on the amount of staff time spent retrieving and re-shelving hard-copy theses;

- there is likely to be a reduction in the number of inter-library loan requests;

- the collection of usage statistics is easier; and

- the provision of an improved service to users should lead to improved levels of customer satisfaction.

\section{Concerns}

A number of areas of concern still exist with regard to the establishment of ETD repositories. For example, advice on copyright and Intellectual Property Rights (IPR) is constantly sought by those involved in the creation and promotion of ETDs. Useful information is available from the 'Creative Commons' Web site (18), but where students wish to reproduce work created by others, or to include video footage of individuals etc., permission to use the material may have to be acquired from a wide range of sources. Further work is needed on these issues.

Authentication is another area where serious consideration has to be given to the policies and procedures adopted: the possible advantages of watermarking, 
timestamping and using digital signatures need to be considered. Various project teams are undertaking work in these areas and it is essential that their conclusions and recommendations are publicised so that others responsible for promoting ETDs and managing institutional repositories may benefit from their findings.

The long term preservation of ETDs which have no printed equivalent is a matter of major significance to libraries. However, the problem of preserving digital material is not unique to ETDs and the establishment of a national collection will ensure that account is taken of theses when developments and agreements in this area take place at national or international level. In the short term, at institutional level, the process of creating and updating regulations to allow the submission of ETDs should involve a debate about acceptable formats and may lead to an improvement on the current practice whereby students often include miscellaneous disks and CD ROM in pockets at the back of printed theses.

An issue of potential concern for both research students and supervisors is the level of I.T. skills required to produce an ETD. At the simplest level, the student may just produce the text in 'Word' and convert the end result into PDF. At a more complex level, the thesis may contain audio material, visual images, video links and hyperlinks. It may not even read sequentially. Institutional guidelines for the submission of ETDs need to identify the people from whom the student must obtain approval for the method they intend to use to convey their research results. Universities need to ensure that adequate equipment and facilities are available to enable students to scan images etc. in order to include non-text elements in their work. Most importantly, those responsible for establishing ETD repositories need to provide adequate training for research students, supervisors and, if necessary, examiners, to ensure that all of the individuals involved in the process are fully conversant with the I.T. being used. 


\section{TECHNICAL ISSUES}

On the technical side, a vast amount of work is being undertaken internationally to improve systems, streamline workflows, and create new opportunities to improve services. Those seeking to establish an institutional repository may consider the selection of appropriate software for this specific purpose the most significant issue.

At The Robert Gordon University (RGU), work undertaken as part of the Electronic Theses project included researching and testing a number of ETD software packages. To determine the most suitable software for the purpose, a set of criteria was identified in order to allow the team to compare and contrast the packages. Initial decisions were based upon written specifications. The preferred software packages were then installed and tested.

\section{Selection of software and related issues at RGU}

\section{Initial requirements}

At the time the Electronic Theses project began at RGU, various potentially suitable software systems were available. The process of selection was made more difficult by the fact that developmental work was still ongoing. The project gave consideration to a number of systems that have already been tested and adopted by respected institutions: DSpace (19), EPrints (20), Virginia Tech. ETD-db (21), Greenstone (22) and several other software packages identified in the Open Society Institute publication 'A Guide to Institutional Repository Software' (23). Many of the packages showed some degree of similarity. The key factor was to select one or more that would be most appropriate for the particular needs of the project. 
The RGU project identified four main factors to which an institution should give consideration when choosing software and setting up an ETD repository:

- Suitability

- Functionality

- Interoperability

- Sustainability

By giving due regard to these points an institution is better placed to set up a successful and manageable archive that will fulfil the needs of the institution and be actively populated for many years ahead.

\section{Suitability}

The software selected should be relatively simple to install across a range of hardware types and operating systems e.g. UNIX-, Linux- and Solaris-based systems. The software should be available at no cost via an open source licence and it should be fully customisable allowing for feature enhancing revisions and upgrades to resolve bugs and possible conflicts.

\section{Functionality}

Much of the desired functionality will remain similar from institution to institution. The following are some of the features that are likely to be required by all:

- An intuitive and appealing user interface for both administration and author purposes - it is important that the system offers a clear and intuitive interface to ensure that authors are encouraged to submit content.

- Long term preservation - the incorporation of features such as persistent URLs to ensure accessibility and future proofing of hyperlinks etc. 
- Searchability - simple and advanced metadata search capabilities allowing for a wide variety of search methods, ideally with full content searching ability.

- Metadata - suitable fields should exist making it possible to alter metadata and thus conform to a national or institutional schema.

- Storage capability - consideration of which file formats and file sizes are to be supported. With the potential for a fully fledged multimedia thesis or document to consist of a variety of formats and span several CDs with information, it is important that restrictions in this area are minimised.

- Security - use of SSL, or other, methods of security.

\section{Interoperability}

To ensure cross institutional access, the software system selected should comply with the most up to date version of the 'Open Archives Protocol for Metadata Harvesting' (OAI-PMH).

The selected software should also meet the needs of individual institutional policies with regard to the integration of ETDs with other material in electronic repositories. This is an important factor to consider to ensure that the new system will be able to offer the functionality to import and export information from one system to another. DSpace's Java API is a good example of how a repository may be enhanced to integrate with other systems. This API layer offers a means whereby the software may be edited by an institution to tailor it to its needs, such as the integration of related software packages currently used in libraries and/or authorisation and verification systems.

\section{Sustainability}

Repositories should be regarded as long term commitments, and thus it is important that the institution can be confident the chosen software will offer 
continued support and development. This is especially important considering the relative infancy of some of the software, where bugs and conflict issues may still be coming to light.

Mailing lists and concise WebPages with online documentation are a significant advantage here. Where these services are offered an ethos of sharing information and experience is promoted and specific problems can be addressed in an informal manner. As is common with much open source software, once a user community is established this knowledge base can help to 'keep the ball rolling' by offering advice and support to those new in the area.

\section{The RGU project selection}

Given the above criteria, and the specific needs of the model being proposed by RGU, the Electronic Theses project has opted to use DSpace and EPrints software for further testing. Both pieces of software satisfy each of the above factors and have been successfully utilised on an international basis. Whilst both these packages are based upon different software technologies, they both have appropriate and broadly similar functionality.

\section{EPrints}

EPrints repository software was developed at the University of Southampton and first made available in 2000. Supported by an active user base, and with the capability of being modified to meet local requirements, EPrints is well suited for an institutional ETD collection. The main technologies behind EPrints are MySQL, Apache and mod_Perl.

DSpace 
DSpace is a digital repository software system designed jointly by MIT Libraries and the Hewlett-Packard Company. Its focus on the submission, storage, access and preservation of research material in digital format makes it well suited to the needs of an institutional ETD collection. The main components required for an installation of DSpace are Java 1.3+, Tomcat 4.0+ and/or Apache 1.3, PostgreSQL 7.3+ and Ant 1.5. Each of these components is freely available and well documented via open source.

An interesting and useful feature of DSpace is the support and inclusion of the handle server. Once registered, this can ensure that each document has a unique and persistent URL. This is a very important point in relation to the long-term preservation of electronic documents, particularly if a paper copy is not easily obtainable. DSpace also captures metadata to assist with digital preservation such as those relating to file format. In addition, file formats are categorised as either supported (where the formats will be functionally preserved using either format migration or emulation), known (popular formats where third party migration tools are likely to emerge) and unsupported (proprietary or unique formats).

\section{Beyond ETDs}

Instead of using these systems to hold ETDs exclusively, the Electronic Theses project is investigating their ability to cope with a range of documents and research output and to be used as the foundation for a Virtual Research Environment (VRE). DSpace and EPrints software appear well suited to this task since a repository based upon a communities and collections structure provides an appropriate layout. As neither system has a restriction on the content that may be submitted, each is capable of storing various types of institutional output - from journal articles and research reports to internal departmental documents. 
The administration and workflow capabilities of the software also make them suitable for the broader purpose.

The Electronic Theses project will investigate this area in more detail over the coming months. Particular attention will be paid towards security, authentication and verification issues as some of the content stored may be sensitive. When building the VRE, the project team will take advantage of the experience gained by other relevant JISC funded projects: HaIRST (Harvesting Institutional Resources in Scotland Testbed) which is based at the University of Strathclyde, SHERPA (Securing a Hybrid Environment for Research Preservation and Access) which is based at the University of Nottingham, E-Prints UK which is based at UKOLN at the University of Bath, and TARDis (Targeting Academic Research for Dissemination and Disclosure) which is based at the University of Southampton.

Whichever approach the Electronic Theses project follows, the intended end result is to promote UK theses and the research work being undertaken within UK Higher Education institutions, to improve ease of access to the full text of theses, and to contribute to the international effort to develop scholarly communication.

The Electronic Theses project aims to encourage all universities in the UK to consider the deposit of full text theses and dissertations in an ETD repository as an integral part of their research process. In most cases this action would involve the creation of an institutional repository; one which may contain only theses but would be more likely to incorporate an ETD collection alongside e-prints and other academic output within a broader 'Virtual Research Environment'. To ensure consistency with regard to access and preservation it would be beneficial if the electronic theses are also included in a national ETD 'hub'. Most theses may be available to researchers in either printed or electronic format initially, but as researchers experience the benefits of ETDs, complete with direct links to primary 
data, it is likely that an increasing number of theses will be available only in electronic format in future years.

References:

1. http://scholar.lib.vt.edu/theses/data/somefacts.html

2. http://www.ndltd.org/

3. ROBERTS, A. Survey on the use of doctoral theses in British universities. Edinburgh: Edinburgh University Library, 1997. (British Library Research and Innovation Report 57). http://libcd2.lib.ed.ac.uk/Theses

4. http://www.unesco.org/webworld/news/000616_etd.shtml

5. http://etdguide.org/

6. http://www.hu-berlin.de/etd2003/

7. http://www.uky.edu/ETD/ETD2004/

8. http://www.cybertheses.org/

9. http://adt.caul.edu.au/

10. http://www.tdx.cesca.es/

11. http://edoc.hu-berlin.de/etd2003/bakelli-yahia/PDF/index.pdf

12. http://dochost.rz.hu-berlin.de/epdiss/index_en.html

13. http://www.jisc.ac.uk/index.cfm?name=programme_fair

14. http://www.openarchives.org/

15. http://www.lib.gla.ac.uk/daedalus/

16. http://www.thesesalive.ac.uk/

17. http://www.rgu.ac.uk/library/e-theses.htm

18. http://creativecommons.org/

19. http://www.dspace.org/

20. http://www.eprints.org/

21. http://scholar.lib.vt.edu/ETD-db/

22. http://www.greenstone.org/

23. http://www.soros.org/openaccess//software/ 